\title{
An Iterative Regularization Method to Solve the Cauchy Problem for the Helmholtz Equation
}

\author{
Hao Cheng, Ping Zhu, and Jie Gao \\ School of Science, Jiangnan University, Jiangsu Province, Wuxi 214122, China \\ Correspondence should be addressed to Hao Cheng; chenghao@jiangnan.edu.cn \\ Received 12 January 2014; Accepted 19 February 2014; Published 25 March 2014 \\ Academic Editor: Rolf Stenberg
}

Copyright (C) 2014 Hao Cheng et al. This is an open access article distributed under the Creative Commons Attribution License, which permits unrestricted use, distribution, and reproduction in any medium, provided the original work is properly cited.

\begin{abstract}
A regularization method for solving the Cauchy problem of the Helmholtz equation is proposed. The $a$ priori and $a$ posteriori rules for choosing regularization parameters with corresponding error estimates between the exact solution and its approximation are also given. The numerical example shows the effectiveness of this method.
\end{abstract}

\section{Introduction}

The Cauchy problem for the Helmholtz equation arises naturally in many areas of engineering and science, especially in wave propagation and vibration phenomena, such as the vibration of a structure [1], the acoustic cavity problem [2], the radiation wave [3], and the scattering of a wave [4]. However, this problem is severely ill-posed in the sense that a small change in the Cauchy data would lead to a dramatic variation in the solution. Therefore, it is necessary to study different highly efficient algorithms to solve this problem. Recently, a few special numerical methods to deal with this problem have been developed, such as the boundary element method [5], the method of fundamental solutions [6], the conjugate gradient method [7], the Landweber method [8], wavelet moment method [9], quasi-reversibility and truncation methods [10], modified Tikhonov regularization method [11, 12], the fourier regularization method [13], and so forth $[9,14,15]$. However, most of them choose the regularization parameter by the $a$ priori rule, which depends seriously on the a priori bound $E$. However, in general, the a priori bound $E$ cannot be known exactly in practice, and working with a wrong constant $E$ may lead to a bad regularized solution. Therefore, giving the a posteriori parameter choice rule is a very meaningful topic.
In this paper we will consider the following problem with inhomogeneous Dirichlet data in a strip domain:

$$
\begin{gathered}
\Delta u(x, y)+k^{2} u(x, y)=0, \quad x \in(0,1), y \in \mathbb{R}, \\
u(0, y)=\varphi(y), \quad y \in \mathbb{R}, \\
u_{x}(0, y)=0, \quad y \in \mathbb{R},
\end{gathered}
$$

where the constant $k>0$ is the number of wave. The solution $u(x, y)$ for $0<x<1$ will be determined from the noisy data $\varphi^{\delta}(y)$. In this paper a regularization method of iteration type for solving this problem will be given. By dint of this method, the $a$ priori and a posteriori rule for choosing a regularization parameter with strict theory analysis, as well as order optimal error estimates, will be obtained.

The outline of the paper is as follows. In Section 2, an order optimal error estimate is obtained for the a priori parameter choice rule. The a posteriori parameter choice rule is given in Section 3, which also leads to a Hölder-type error estimate. Numerical implement shows the effectiveness of the proposed method in Section 4.

\section{Regularization and Error Estimate}

Let $\widehat{g}(\xi)$ denote the Fourier transform of the function $g(y)$, which is defined as

$$
\widehat{g}(\xi)=\frac{1}{\sqrt{2 \pi}} \int_{-\infty}^{\infty} e^{-i y \xi} g(y) d y, \quad i=\sqrt{-1} .
$$


The functions $\varphi(y), \varphi^{\delta}(y) \in L^{2}(\mathbb{R})$ are the exact and measured data for problem (1), respectively, and satisfy

$$
\left\|\varphi^{\delta}(\cdot)-\varphi(\cdot)\right\| \leq \delta
$$

where $\|\cdot\|$ denotes the $L^{2}$-norm and the constant $\delta>0$ is the noise level. Assume that $u(x, \cdot) \in L^{2}(\mathbb{R})$ for all $0 \leq x<1$ and there is the following a priori bound;

$$
\|u(1, \cdot)\| \leq E,
$$

where $E$ is a positive constant.

It is easy to know that for problem (1),

$$
\widehat{u}(x, \xi)=\cosh \left(x \sqrt{|\xi|^{2}-k^{2}}\right) \widehat{\varphi}(\xi),
$$

and equivalently,

$$
u(x, y)=\frac{1}{\sqrt{2 \pi}} \int_{-\infty}^{\infty} e^{i y \xi} \cosh \left(x \sqrt{|\xi|^{2}-k^{2}}\right) \hat{\varphi}(\xi) d \xi .
$$

Note that the factor $\cosh \left(x \sqrt{|\xi|^{2}-k^{2}}\right)$ increases exponentially for $0<x<1$ as $|\xi| \rightarrow+\infty$; a small distribution for the data $\varphi(x)$ will be amplified infinitely by this factor and lead to the integral (6) blow-up. Therefore, recovering the temperature $u(x, y)$ from the measured data $\varphi^{\delta}(x)$ is severely ill-posed.

For simplicity [15], we decompose $\mathbb{R}$ into the following parts $I$ and $W$, where:

$$
I:=\{\xi \in \mathbb{R},|\xi| \geq k\}, \quad W:=\{\xi \in \mathbb{R},|\xi| \leq k\} ;
$$

then $L^{2}(\mathbb{R})=L^{2}(I) \oplus L^{2}(W)$.

For $\xi \in W$, we can take the regularization approximation solution in the frequency domain as

$$
\widehat{u}_{m}^{\delta}(x, \xi)=\cos \left(x \sqrt{k^{2}-|\xi|^{2}}\right) \widehat{\varphi}^{\delta}(\xi) .
$$

For $\xi \in I$, we introduce an iteration scheme with the following form:

$$
\begin{aligned}
\widehat{u}_{m}^{\delta}(x, \xi)= & (1-\lambda) \widehat{u}_{m-1}^{\delta}(x, \xi) \\
& +\lambda \cosh \left(x \sqrt{|\xi|^{2}-k^{2}}\right) \widehat{\varphi}^{\delta}(\xi), \\
& m=1,2, \ldots,
\end{aligned}
$$

where $\lambda=\lambda(\xi)=e^{-\sqrt{|\xi|^{2}-k^{2}}}<1$ plays an important role in the convergence proof; the initial guess is $\widehat{u}_{0}^{\delta}(x, \xi)$. By using an elementary calculation for (9), we obtain

$$
\begin{aligned}
\widehat{u}_{m}^{\delta}(x, \xi)= & (1-\lambda)^{m} \widehat{u}_{0}^{\delta}(x, \xi) \\
& +\sum_{i=0}^{m-1}(1-\lambda)^{i} \lambda \cosh \left(x \sqrt{|\xi|^{2}-k^{2}}\right) \widehat{\varphi}^{\delta}(\xi) \\
= & (1-\lambda)^{m} \widehat{u}_{0}^{\delta}(x, \xi)+\left(1-(1-\lambda)^{m}\right) \\
& \times \cosh \left(x \sqrt{|\xi|^{2}-k^{2}}\right) \widehat{\varphi}^{\delta}(\xi) .
\end{aligned}
$$

Therefore, the approximate solution of problem (1) has the following form in the frequency domain:

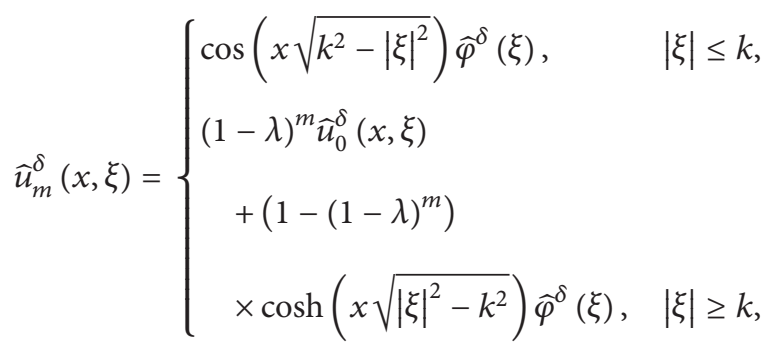

or equivalently,

$$
u_{m}^{\delta}(x, y)=\frac{1}{\sqrt{2 \pi}} \int_{-\infty}^{\infty} e^{i x \xi} \widehat{u}_{m}^{\delta}(x, \xi) d \xi
$$

where $\widehat{u}_{m}^{\delta}(x, \xi)$ is given by (11).

Lemma 1 (see [16]). For $0<\lambda \leq 1$ and $m \geq 1$, the following inequalities hold:

$$
\begin{aligned}
& (1-\lambda)^{m} \lambda \leq \frac{1}{m+1}, \\
& \frac{1-(1-\lambda)^{m}}{\lambda} \leq m .
\end{aligned}
$$

Lemma 2. For $0 \leq \lambda \leq 1,0 \leq \alpha \leq 1$ and $m \geq 1$, Lemma 1 can be strengthened as the following inequalities:

$$
\begin{gathered}
(1-\lambda)^{m} \lambda^{\alpha} \leq(m+1)^{-\alpha}, \\
\frac{1-(1-\lambda)^{m}}{\lambda^{\alpha}} \leq m^{\alpha} .
\end{gathered}
$$

Proof. In fact, using the established results (13), we can get

$$
\begin{aligned}
& (1-\lambda)^{m} \lambda^{\alpha} \leq\left[(1-\lambda)^{m} \lambda\right]^{\alpha} \leq(m+1)^{-\alpha}, \\
& \frac{1-(1-\lambda)^{m}}{\lambda^{\alpha}} \leq\left[\frac{1-(1-\lambda)^{m}}{\lambda}\right]^{\alpha} \leq m^{\alpha} .
\end{aligned}
$$

Theorem 3. Let $u(x, y)$ be the exact solution of problem (1) and $u_{m}^{\delta}(x, y)$ be its regularized approximation given by (12) with $u_{0}^{\delta}(x, y)=0$. Assumptions (3) and (4) are satisfied and one chooses $m=\lfloor E / \delta\rfloor$, where $\lfloor t\rfloor$ denotes the largest integer not exceeding $t$; then there holds the following estimate:

$$
\left\|u(x, \cdot)-u_{m}^{\delta}(x, \cdot)\right\| \leq 3 E^{x} \delta^{1-x}+\delta, \quad 0<x<1 .
$$

Proof. Due to the Parseval formula and the triangle inequality, we have

$$
\begin{aligned}
\left\|u(x, \cdot)-u_{m}^{\delta}(x, \cdot)\right\|^{2}= & \left\|\widehat{u}(x, \cdot)-\widehat{u}_{m}^{\delta}(x, \cdot)\right\|^{2} \\
= & \left\|\widehat{u}(x, \cdot)-\widehat{u}_{m}^{\delta}(x, \cdot)\right\|_{L^{2}(W)}^{2} \\
& +\left\|\widehat{u}(x, \cdot)-\widehat{u}_{m}^{\delta}(x, \cdot)\right\|_{L^{2}(I)}^{2} .
\end{aligned}
$$


Case 1. While $\xi \in W$, combining (3), (6), and (12), we have

$$
\begin{aligned}
& \left\|\widehat{u}(x, \cdot)-\widehat{u}_{m}^{\delta}(x, \cdot)\right\|_{L^{2}(W)} \\
& =\| \cos \left(x \sqrt{k^{2}-|\xi|^{2}}\right) \widehat{\varphi}(\xi) \\
& \quad-\cos \left(x \sqrt{k^{2}-|\xi|^{2}}\right) \widehat{\varphi}^{\delta}(\xi) \|_{L^{2}(W)} \\
& \leq\left\|\widehat{\varphi}(\xi)-\widehat{\varphi}^{\delta}(\xi)\right\|_{L^{2}(W)} \\
& \leq\left\|\widehat{\varphi}(\xi)-\widehat{\varphi}^{\delta}(\xi)\right\|^{\leq} \\
& \leq \delta .
\end{aligned}
$$

Case 2. For $\xi \in I$, combining (4), (6), (12), (14), and (15), we have

$$
\begin{aligned}
& \left\|\widehat{u}(x, \cdot)-\widehat{u}_{m}^{\delta}(x, \cdot)\right\|_{L^{2}(I)} \\
& =\| \cosh \left(x \sqrt{|\xi|^{2}-k^{2}}\right) \widehat{\varphi} \\
& -\cosh \left(x \sqrt{|\xi|^{2}-k^{2}}\right)\left(1-(1-\lambda)^{m}\right) \hat{\varphi}^{\delta} \| \\
& \leq\left\|\cosh \left(x \sqrt{|\xi|^{2}-k^{2}}\right)(1-\lambda)^{m} \hat{\varphi}\right\| \\
& +\left\|\cosh \left(x \sqrt{|\xi|^{2}-k^{2}}\right)\left(1-(1-\lambda)^{m}\right)\left(\widehat{\varphi}-\widehat{\varphi}^{\delta}\right)\right\| \\
& =\left\|\frac{\cosh \left(x \sqrt{|\xi|^{2}-k^{2}}\right)(1-\lambda)^{m}}{\cosh \left(\sqrt{|\xi|^{2}-k^{2}}\right)} \cosh \left(\sqrt{|\xi|^{2}-k^{2}}\right) \hat{\varphi}\right\| \\
& +\left\|\cosh \left(x \sqrt{|\xi|^{2}-k^{2}}\right)\left(1-(1-\lambda)^{m}\right)\left(\hat{\varphi}^{\delta}-\widehat{\varphi}\right)\right\| \\
& \leq E \sup _{\xi \in \mathbb{R}} \frac{\cosh \left(x \sqrt{|\xi|^{2}-k^{2}}\right)(1-\lambda)^{m}}{\cosh \left(\sqrt{|\xi|^{2}-k^{2}}\right)} \\
& +\delta \sup _{\xi \in \mathbb{R}} \cosh \left(x \sqrt{|\xi|^{2}-k^{2}}\right)\left(1-(1-\lambda)^{m}\right) \\
& \leq 2 E \sup _{\xi \in \mathbb{R}}(1-\lambda)^{m} e^{(x-1) \sqrt{|\xi|^{2}-k^{2}}} \\
& +\delta \sup _{\xi \in \mathbb{R}}\left(1-(1-\lambda)^{m}\right) e^{x \sqrt{|\xi|^{2}-k^{2}}} \\
& =2 E \sup _{0<\lambda<1} \lambda^{(1-x)}(1-\lambda)^{m} \\
& +\delta \sup _{0<\lambda<1} \lambda^{-x}\left(1-(1-\lambda)^{m}\right) \\
& \leq 2 E(m+1)^{x-1}+\delta m^{x} .
\end{aligned}
$$

Due to $m=\lfloor E / \delta\rfloor$, then $m \leq E / \delta$ and $m+1 \geq E / \delta$, therefore,

$$
\begin{aligned}
\left\|\widehat{u}(x, \cdot)-\widehat{u}_{m}^{\delta}(x, \cdot)\right\|_{L^{2}(I)} & \leq 2 E\left(\frac{E}{\delta}\right)^{x-1}+\delta\left(\frac{E}{\delta}\right)^{x} \\
& =3 E^{x} \delta^{1-x} .
\end{aligned}
$$

Combing inequalities (18), (19), and (21), the proof of this theorem is completed.

Remark 4. Obviously, Theorem 3 could only solve the problem with the case $0<x<1$. The stronger smoothness assumption of $\|u(1, \cdot)\|$ may obtain convergence rates for the endpoint $x=1$; see, for example, [10-12], and we omit the further discussions.

\section{The Discrepancy Principle}

In this section, we discuss an a posteriori stopping rule for iterative scheme (9) which is based on the discrepancy principle of Morozov [17, 18] in the following form:

$$
\left\|\varphi^{\delta}-u_{m}^{\delta}(0, \cdot)\right\| \leq \tau \delta \leq\left\|\varphi^{\delta}-u_{m-1}^{\delta}(0, \cdot)\right\|,
$$

where $\tau>1$ is a constant and $m$ denotes the regularization parameter. In the numerical experiments, we can take the iteration depth $m$ which satisfies (22) firstly.

If $\widehat{u}_{0}^{\delta}(x, \xi)=0$, thus (22) can be simplified to

$$
\left\|(1-\lambda)^{m} \widehat{\varphi}^{\delta}\right\|_{L^{2}(I)} \leq \tau \delta \leq\left\|(1-\lambda)^{m-1} \widehat{\varphi}^{\delta}\right\|_{L^{2}(I)} .
$$

Lemma 5. The following inequality holds:

$$
m \leq \frac{2 E}{(\tau-1) \delta} .
$$

Proof. Due to (4) and (14), we know

$$
\begin{aligned}
\tau \delta & \leq\left\|\varphi^{\delta}-u_{m-1}^{\delta}(0, \cdot)\right\|=\left\|(1-\lambda)^{m-1} \widehat{\varphi}^{\delta}\right\|_{L^{2}(I)} \\
& \leq\left\|(1-\lambda)^{m-1}\left(\widehat{\varphi}^{\delta}-\widehat{\varphi}\right)\right\|_{L^{2}(I)}+\left\|(1-\lambda)^{m-1} \widehat{\varphi}\right\|_{L^{2}(I)} \\
& \leq\left\|\widehat{\varphi}^{\delta}-\hat{\varphi}\right\|_{L^{2}(I)}+\left\|(1-\lambda)^{m-1} \frac{\cosh \left(\sqrt{|\xi|^{2}-k^{2}}\right) \hat{\varphi}}{\cosh \left(\sqrt{|\xi|^{2}-k^{2}}\right)}\right\|_{L^{2}(I)} \\
& \leq \delta+2 E \sup _{0<\lambda<1}(1-\lambda)^{m-1} \lambda \\
& \leq \delta+\frac{2 E}{m},
\end{aligned}
$$

therefore,

$$
m \leq \frac{2 E}{(\tau-1) \delta}
$$

Lemma 6. Setting $\omega_{m}(x, \cdot)=u(x, \cdot)-u_{m}(x, \cdot)$, then the following inequality holds:

$$
\left\|\omega_{m}(x, \cdot)\right\|_{L^{2}(I)} \leq 2\left\|\omega_{m}(1, \cdot)\right\|_{L^{2}(I)}^{x}\left\|\omega_{m}(0, \cdot)\right\|_{L^{2}(I)}^{1-x} .
$$


Proof. Defining $u_{m}(x, \cdot)=\left(1-(1-\lambda)^{m}\right) \cosh \left(x \sqrt{|\xi|^{2}-k^{2}}\right)$ $\widehat{\varphi}(\xi)$, then we have

$$
\begin{aligned}
& \omega_{m}(0, \cdot)=(1-\lambda)^{m} \hat{\varphi}, \\
& \omega_{m}(1, \cdot)=(1-\lambda)^{m} \cosh \left(\sqrt{|\xi|^{2}-k^{2}}\right) \widehat{\varphi} \text {. } \\
& \left\|\omega_{m}(x, \cdot)\right\|_{L^{2}(I)}^{2} \\
& =\left\|(1-\lambda)^{m} \cosh \left(x \sqrt{|\xi|^{2}-k^{2}}\right) \hat{\varphi}\right\|_{L^{2}(I)}^{2} \\
& =\int_{\xi \in I}\left(1-e^{-\sqrt{|\xi|^{2}-k^{2}}}\right)^{2 m}\left(\cosh \left(x \sqrt{|\xi|^{2}-k^{2}}\right) \varphi\right)^{2} d \xi \\
& \leq \int_{\xi \in I}\left(1-e^{-\sqrt{|\xi|^{2}-k^{2}}}\right)^{2 m}\left(e^{x \sqrt{|\xi|^{2}-k^{2}}} \varphi\right)^{2} d \xi \\
& =\int_{\xi \in I}\left(1-e^{\sqrt{|\xi|^{2}-k^{2}}}\right)^{2 m x}\left(e^{\sqrt{|\xi|^{2}-k^{2}}} \varphi\right)^{2 x} \\
& \times\left(1-e^{\sqrt{|\xi|^{2}-k^{2}}}\right)^{2 m(1-x)}\left(e^{\sqrt{|\xi|^{2}-k^{2}}} \varphi\right)^{2(1-x)} d \xi \\
& \leq\left(\int_{\xi \in I}\left(\left(1-e^{-\sqrt{|\xi|^{2}-k^{2}}}\right)^{m} e^{\sqrt{|\xi|^{2}-k^{2}}} \varphi\right)^{2} d \xi\right)^{x} \\
& \times\left(\int_{\xi \in I}\left(\left(1-e^{-\sqrt{|\xi|^{2}-k^{2}}}\right)^{m} \varphi\right)^{2} d \xi\right)^{1-x} \\
& \leq 4\left(\int_{\xi \in I}\left(\left(1-e^{-\sqrt{|\xi|^{2}-k^{2}}}\right)^{m} \cosh \left(\sqrt{|\xi|^{2}-k^{2}}\right) \varphi\right)^{2} d \xi\right)^{x} \\
& \times\left(\int_{\xi \in I}\left(\left(1-e^{-\sqrt{|\xi|^{2}-k^{2}}}\right)^{m} \varphi\right)^{2} d \xi\right)^{1-x} \\
& =4\left\|\omega_{m}(1, \cdot)\right\|_{L^{2}(I)}^{2 x}\left\|\omega_{m}(0, \cdot)\right\|_{L^{2}(I)}^{2(1-x)} .
\end{aligned}
$$

Lemma 7. The following inequality holds:

$$
\left\|u_{m}(x, \cdot)-u(x, \cdot)\right\|_{L^{2}(I)} \leq 2(\tau+1)^{1-x} E^{x} \delta^{1-x} .
$$

Proof. Due to (3), (4), and (27), we know that

$$
\begin{aligned}
& \left\|u_{m}(1, \cdot)-u(1, \cdot)\right\|_{L^{2}(I)} \\
& \quad=\left\|(1-\lambda)^{m} \cosh \left(\sqrt{|\xi|^{2}-k^{2}}\right) \varphi\right\| \leq E, \\
& \left\|u_{m}(0, \cdot)-u(0, \cdot)\right\|_{L^{2}(I)} \\
& \quad=\left\|(1-\lambda)^{m} \varphi\right\|_{L^{2}(I)}
\end{aligned}
$$

$$
\begin{aligned}
\leq & \left\|\left(1-e^{-\sqrt{|\xi|^{2}-k^{2}}}\right)^{m}\left(\varphi-\varphi^{\delta}\right)\right\|_{L^{2}(I)} \\
& +\left\|\left(1-e^{-\sqrt{|\xi|^{2}-k^{2}}}\right)^{m} \varphi^{\delta}\right\|_{L^{2}(I)} \\
& \leq(\tau+1) \delta .
\end{aligned}
$$

Combining (30), we have

$$
\left\|u_{m}(x, \cdot)-u(x, \cdot)\right\|_{L^{2}(I)} \leq 2(\tau+1)^{1-x} E^{x} \delta^{1-x} .
$$

Theorem 8. Let $u(x, y)$ be the exact solution of problem (1) and $u_{m}^{\delta}(x, y)$ be its regularization approximation defined by (12) with $u_{0}^{\delta}(x, y)=0$. If the a priori bound (4) is valid and the iteration (9) is stopped by the discrepancy principle (22), then

$$
\left\|u_{m}^{\delta}(x, \cdot)-u(x, \cdot)\right\| \leq C E^{y / y_{0}} \delta^{1-\left(y / y_{0}\right)}+\delta,
$$

where $C=(2 /(\tau-1))^{x}+2(\tau+1)^{1-x}$.

Proof. According to the triangle inequality, (19), (24), and (29), we obtain that

$$
\begin{aligned}
& \left\|u_{m}^{\delta}(x, \cdot)-u(x, \cdot)\right\| \\
& \quad \leq\left\|u_{m}^{\delta}(x, \cdot)-u(x, \cdot)\right\|_{L^{2}(I)}+\left\|u_{m}^{\delta}(x, \cdot)-u(x, \cdot)\right\|_{L^{2}(W)} \\
& \quad \leq\left\|u_{m}^{\delta}(x, \cdot)-u_{m}(x, \cdot)\right\|_{L^{2}(I)}+\left\|u_{m}(x, \cdot)-u(x, \cdot)\right\|_{L^{2}(I)}+\delta \\
& \quad \leq \delta m^{x}+2(\tau+1)^{1-x} E^{x} \delta^{1-x}+\delta \\
& \quad \leq \delta\left(\frac{2 E}{(\tau-1) \delta}\right)^{x}+2(\tau+1)^{1-x} E^{x} \delta^{1-x}+\delta \\
& \quad=\left(\left(\frac{2}{\tau-1}\right)^{x}+2(\tau+1)^{1-x}\right) E^{x} \delta^{1-x}+\delta .
\end{aligned}
$$

\section{Numerical Test}

In this section, a simple numerical example is devised to verify the validity of the proposed method. We use the discrete Fourier transform and inverse Fourier transform (or FFT and IFFT algorithms) to complete our numerical experiment. We fix the interval $a \leq y \leq b, N$ denotes the number of discrete points.

For an exact data function $\varphi(y)$, its discrete noisy version is

$$
\varphi^{\epsilon}=\varphi+\epsilon \operatorname{rand} n(\operatorname{size}(\varphi))
$$

where

$$
\begin{gathered}
\varphi=\left(\varphi\left(y_{1}\right), \ldots, \varphi\left(y_{N}\right)\right), \\
y_{j}=a+\frac{(b-a)(j-1)}{N+1}, \quad j=1,2 \ldots, N, \\
\delta=\left\|\varphi^{\epsilon}-\varphi\right\|_{l^{2}}:=\sqrt{\frac{1}{N} \sum_{i=1}^{N}\left|\varphi^{\epsilon}\left(y_{i}\right)-\varphi\left(y_{i}\right)\right|^{2} .}
\end{gathered}
$$



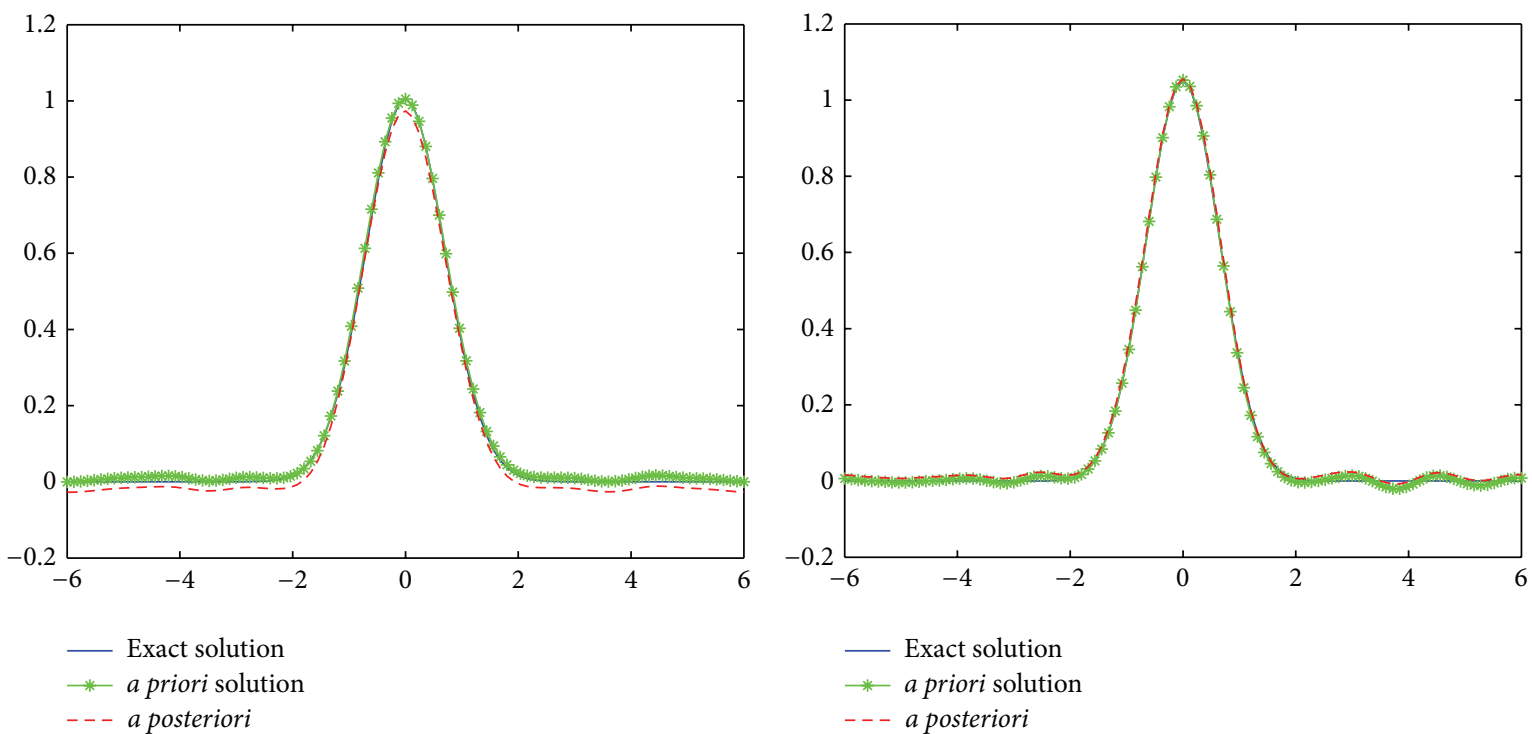

(a)

(b)
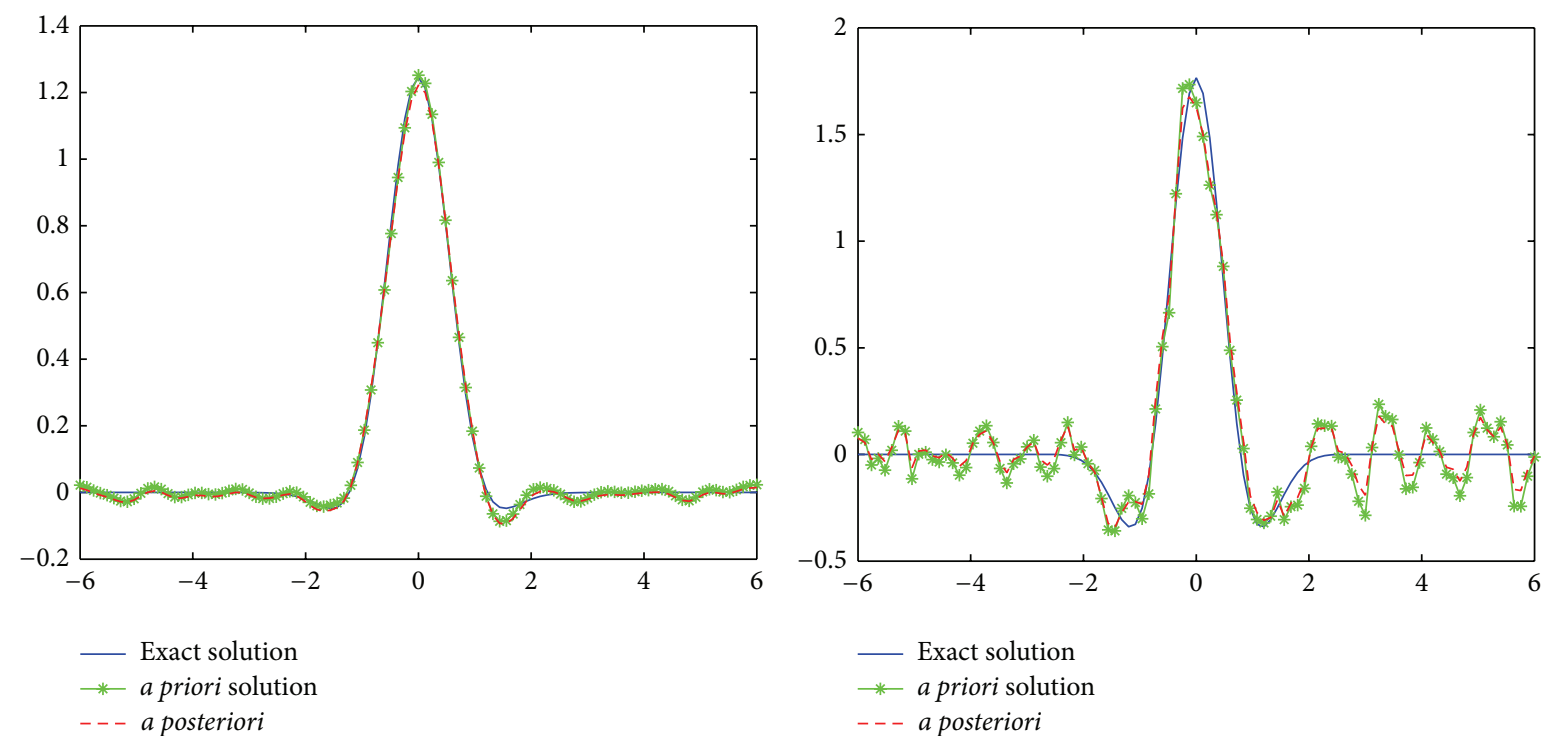

(c)

(d)

FIGURE 1: Example 1. The regularization solution with a priori and a posteriori parameter choice rules for the noise level $\epsilon=10^{-2}$. (a) $y=0.1$, (b) $y=0.3$, (c) $y=0.6$, and (d) $y=0.9$, respectively.

The function "rand $n(\cdot)$ " generates arrays of random numbers whose elements are normally distributed with mean 0 , variance $\sigma^{2}=1$. The absolute error $e_{a}(u)$ and the relative error $e_{r}(u)$ are defined by

$$
\begin{aligned}
& e_{a}(u):=\left\|u_{m}^{\delta}(x, \cdot)-u(x, \cdot)\right\|_{l^{2}}, \\
& e_{r}(u):=\frac{\left\|u_{m}^{\delta}(x, \cdot)-u(x, \cdot)\right\|_{l^{2}}}{\|u(x, \cdot)\|_{l^{2}}},
\end{aligned}
$$

respectively.

In the numerical experiment, we compute the approximation $u_{m}^{\delta}(x, y)$ according to Theorem 3 . And we can take the discrete points $N=100$, the number of wave $k=1$, a priori bound $E=\|u(1, \cdot)\|_{l^{2}(\mathbb{R})} \approx 0.7$, and a priori parameter $m=$ $\lfloor E / \delta\rfloor$. The a posteriori parameter $m$ was chosen according to formula (22) and $\tau=2$ for calculation. Meanwhile, we take $a=-6$ and $b=6$ in the first numerical example. For Example 2, we take $a=-4$ and $b=4$.

Example 9. If we take the function $\varphi(y)=e^{-y^{2}} \in \mathcal{S}(\mathbb{R})$, where $\delta(\mathbb{R})$ denotes the Schwartz function space, $\widehat{\varphi}(\xi) \epsilon$ $\delta(\mathbb{R})$ decays rapidly and formula (6) can be used to calculate $u(x, y)$ with exact data directly. To observe the effect on different noise levels $\epsilon$, we only take the case of $k=1$ at $x=0.9$.

Table 1 shows the comparison of the errors between the exact and regularization solutions for different $\epsilon$, from which 

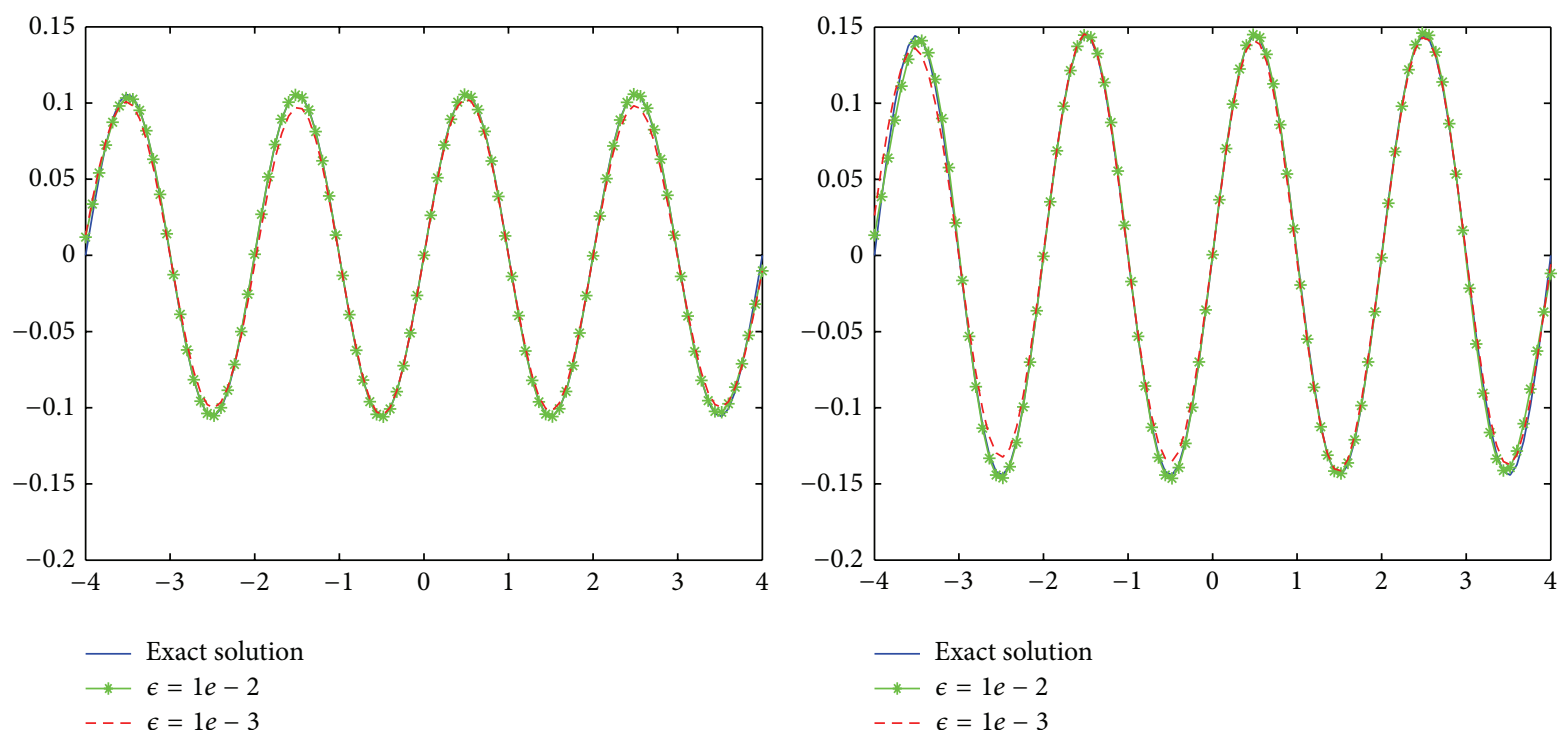

(a)

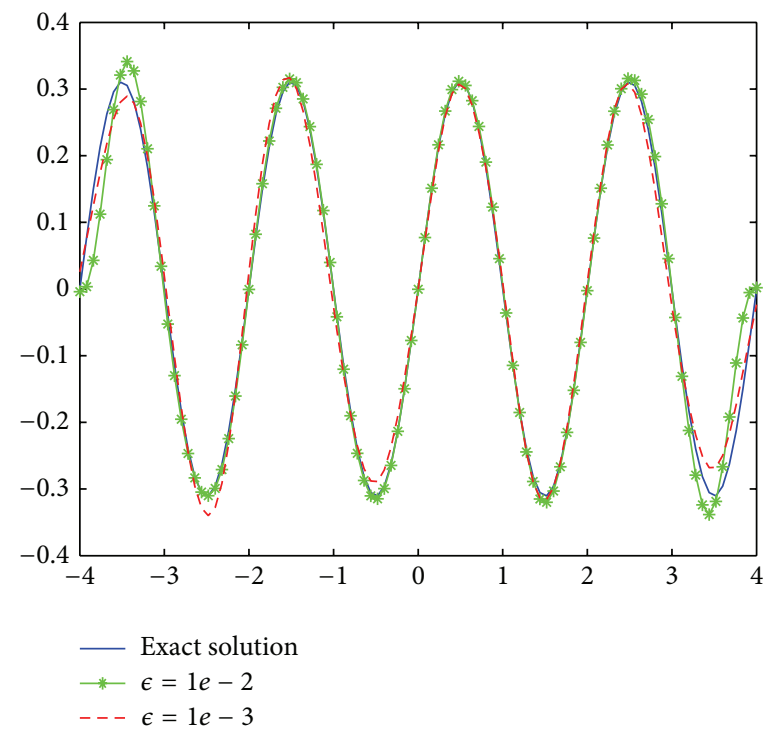

(c)

(b)

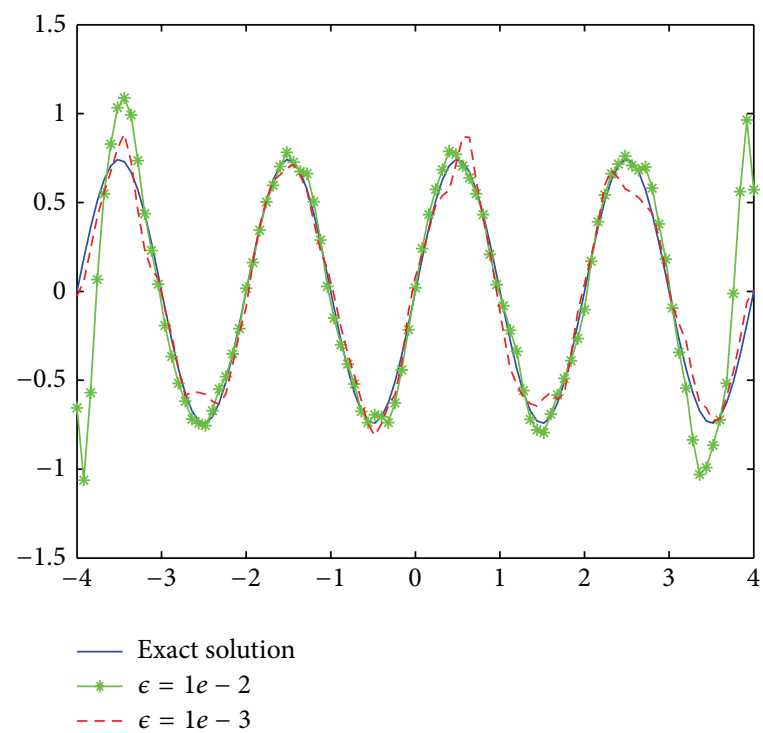

(d)

Figure 2: Example 2. The exact and regularized solutions for the different noise levels. (a) $x=0.1$, (b) $x=0.3$, (c) $x=0.6$, and (d) $x=0.9 \epsilon=10^{-2}, 10^{-3}$, respectively.

TABLE 1: The errors between the exact and approximate solutions of Example 1, with $k=1$ at $x=0.9$.

\begin{tabular}{lcccc}
\hline$\epsilon$ & $1 e-4$ & $1 e-3$ & $1 e-2$ & $1 e-1$ \\
\hline$m$ & 4909 & 534 & 51 & 4 \\
$e_{a}(u)$ & 0.4435 & 0.5194 & 0.8220 & 2.1846 \\
$e_{r}(u)$ & 0.1021 & 0.1196 & 0.1893 & 0.5031 \\
\hline
\end{tabular}

we can see that the smaller the $\epsilon$ is, the better the computed approximation is.

Figure 1 is the comparison of a priori and a posteriori parameter choice rules for the exact $u(x, y)$ and the approximate solution $u_{k}^{\delta}(x, y)$ at $x=0.1,0.3,0.6,0.9$ for the noise level $\epsilon=10^{-2}$. Here we also take the reasonable a priori bound $E=\|u(1, \cdot)\|_{l^{2}(\mathbb{R})}$, and we can see that the a posteriori rule also works effectively.

Example 10. The function

$$
u(x, y)=\frac{1}{\pi^{2}} \sin (\pi k y) \cosh \left(k \sqrt{\pi^{2}-1} x\right)
$$

is the exact solution of problem (1) with the Cauchy data $u(0, y)=\varphi(y)=\left(1 / \pi^{2}\right) \sin (\pi k y)$ and $u_{x}(0, y)=0$.

Figure 2 is the comparison of the exact solution $u(x, y)$ and the approximation $u_{m}^{\delta}(x, y)$ at different points $x=0.1$, $0.3,0.6,0.9$ and noise levels $\epsilon=10^{-2}, 10^{-3}$ for the a priori 


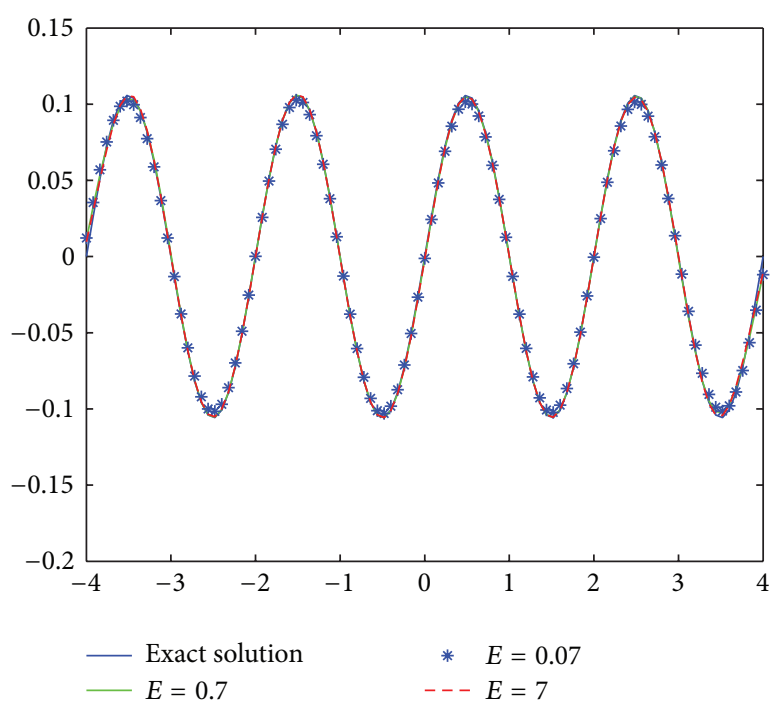

(a)

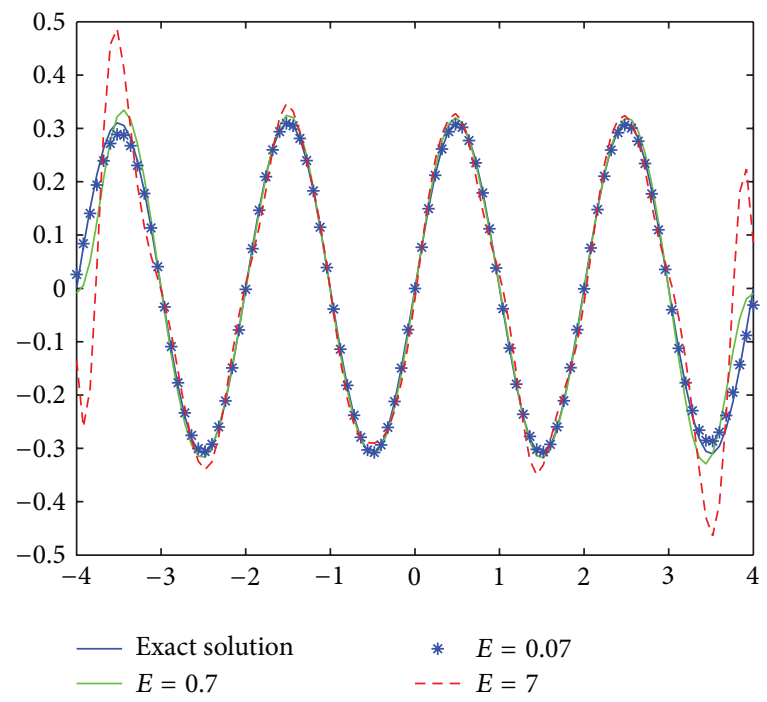

(c)

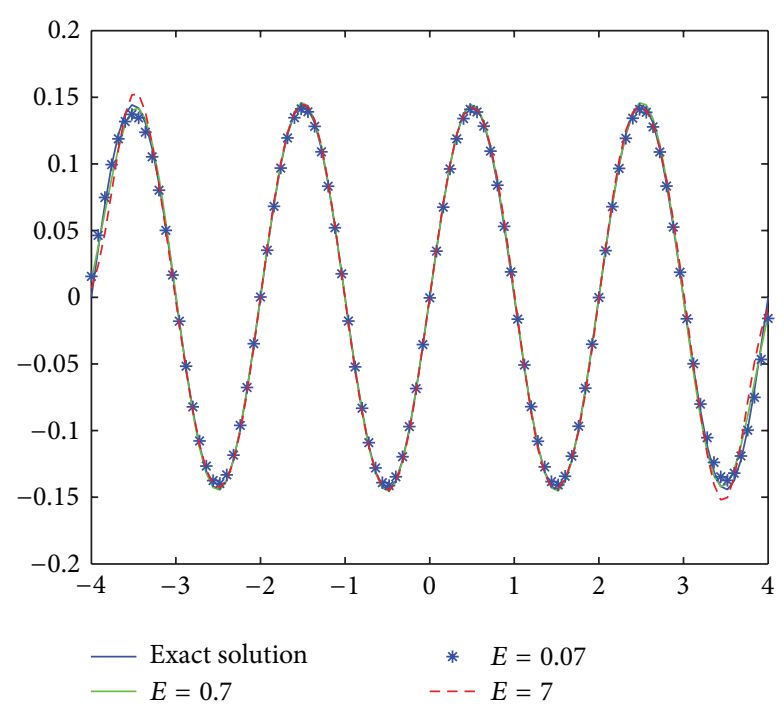

(b)

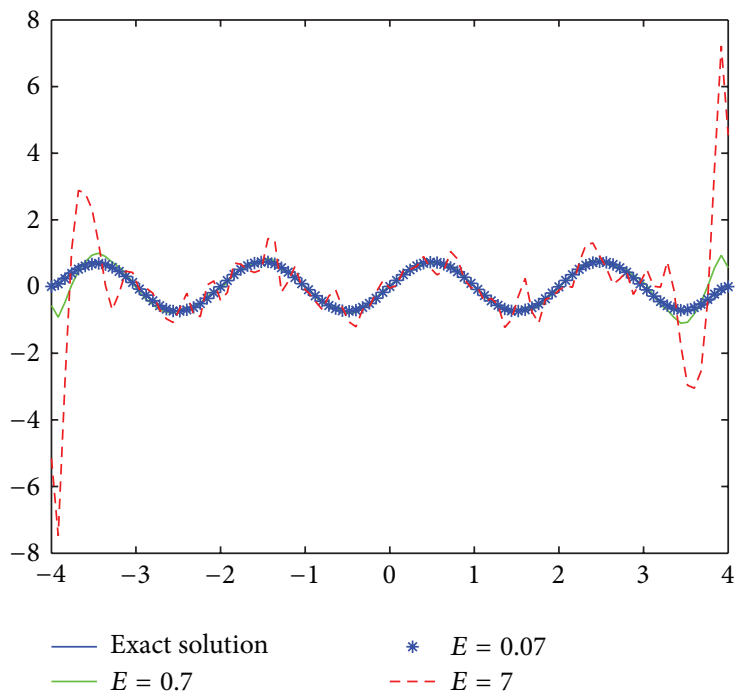

(d)

Figure 3: Example 2. The exact and regularized solutions at (a) $x=0.1$, (b) $x=0.3$, (c) $x=0.6$, and (d) $x=0.9$ for the same noise level $\epsilon=10^{-2}$ but different $a$ priori bounds $E=0.07,0.7,7$, respectively.

parameter choice rule. Here we take the a priori bound $E=$ $\|u(1, \cdot)\|_{l^{2}(\mathbb{R})}$, and the proposed method works well for the $a$ priori parameter choice rule.

Figure 3 is the comparison of the different a priori bound $E=0.07,0.7,7$ for the different points $x=0.1,0.3,0.6,0.9$ at the noise level $\epsilon=10^{-2}$. From this figure we can see that working with a wrong constant $E$ would lead to a bad regularized solution. Therefore, a reasonable a priori bound $E$ is very important for the a priori parameter choice rule.

Figure 4 is the comparison of a priori and a posteriori parameter choice rules for the exact $u(x, y)$ and the approximate solution $u_{m}^{\delta}(x, y)$ at $x=0.1,0.3,0.6,0.9$ for the noise level $\epsilon=10^{-2}$. Here we take the reasonable a priori bound $E=\|u(1, \cdot)\|_{l^{2}(\mathbb{R})}$ as previously mentioned, and the a posteriori rule also works effectively as expected.
From Figures 1-4, we concluded that the smaller the $\epsilon$ is, the better the computed approximation is, and the bigger the $x$ is, the worse the computed approximation is. In addition, the a priori bound $E$ has great influence on the numerical results. Although the a posteriori regularization parameter selection rule does not rely on a priori bound $E$, it also works well.

\section{Conclusion}

In this paper an iteration regularization method is given for solving the numerical analytic continuation problem on a strip domain. The a priori and a posteriori rules for choosing a regularization parameter with strict theory analysis are presented. In numerical aspect, the comparison with different 


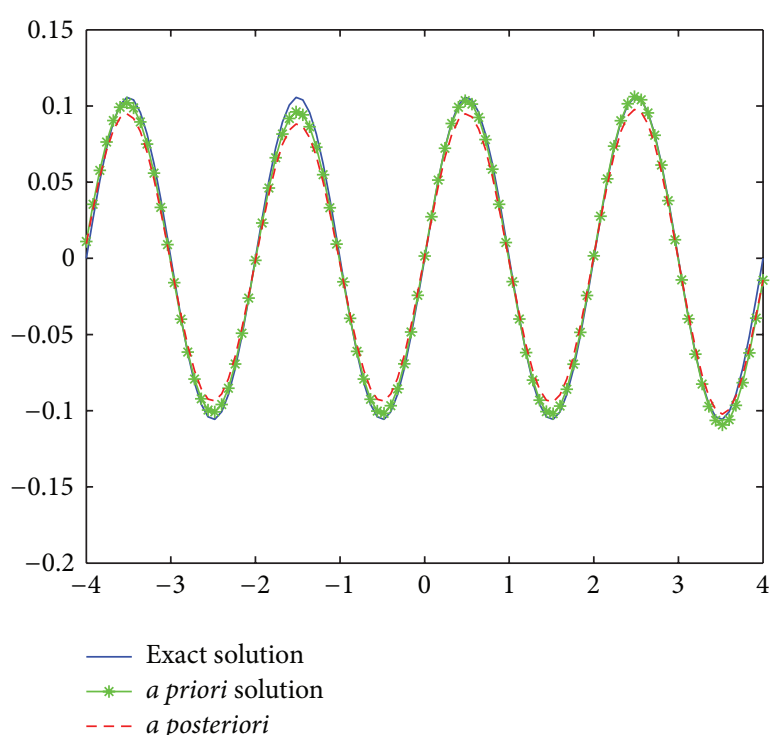

(a)

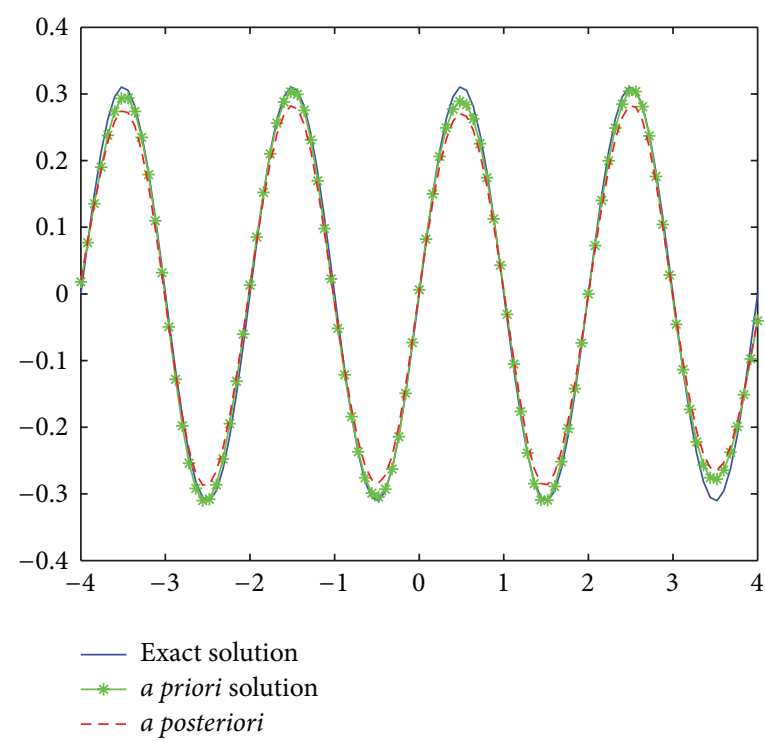

(c)

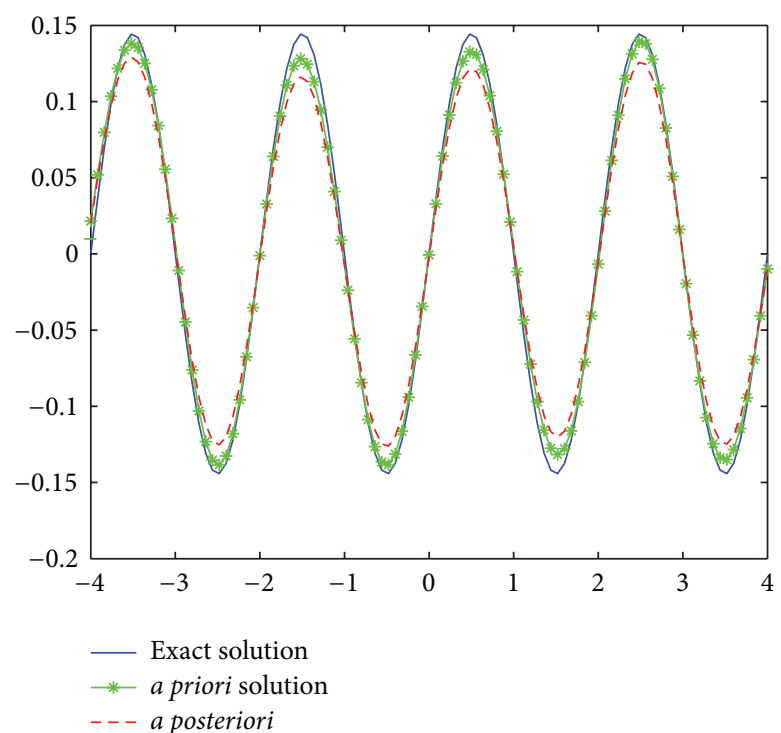

(b)

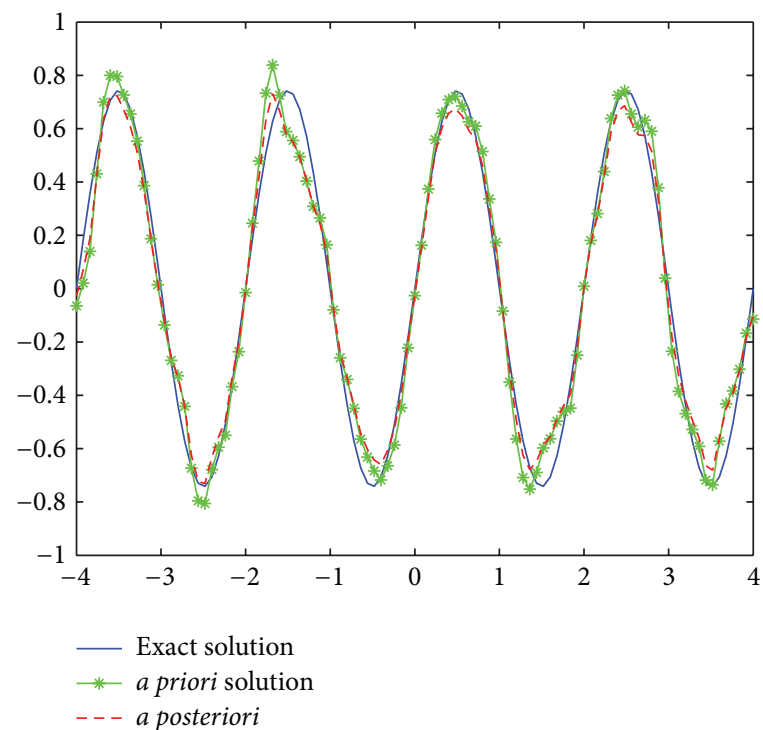

(d)

FIgURE 4: Example 2. The regularization solution with a priori and a posteriori parameter choice rules for the noise level $\epsilon=10^{-2}$. (a) $x=0.1$, (b) $x=0.3$, (c) $x=0.6$, and (d) $x=0.9$, respectively.

parameter choice rules shows that the proposed method works effectively.

\section{Conflict of Interests}

The authors declare that there is no conflict of interests regarding the publication of this paper.

\section{Acknowledgment}

The project is supported by the Natural Science Foundation of Jiangsu Province of China for Young Scholar (no.
BK20130118), the Fundamental Research Funds for the Central Universities (no. JUSRP1033), and the NNSF of China (nos. 11171136, 11271163, and 11371174).

\section{References}

[1] D. E. Beskos, "Boundary element methods in dynamic analysis: part II (1986-1996)," Applied Mechanics Reviews, vol. 50, no. 3, pp. 149-197, 1997.

[2] J. T. Chen and F. C. Wong, "Dual formulation of multiple reciprocity method for the acoustic mode of a cavity with a thin partition," Journal of Sound and Vibration, vol. 217, no. 1, pp. 7595, 1998. 
[3] I. Harari, P. E. Barbone, M. Slavutin, and R. Shalom, "Boundary infinite elements for the Helmholtz equation in exterior domains," International Journal for Numerical Methods in Engineering, vol. 41, no. 6, pp. 1105-1131, 1998.

[4] W. S. Hall and X. Q. Mao, "A boundary element investigation of irregular frequencies in electromagnetic scattering," Engineering Analysis with Boundary Elements, vol. 16, no. 3, pp. 245-252, 1995.

[5] L. Marin, L. Elliott, P. J. Heggs, D. B. Ingham, D. Lesnic, and $\mathrm{X}$. Wen, "An alternating iterative algorithm for the Cauchy problem associated to the Helmholtz equation," Computer Methods in Applied Mechanics and Engineering, vol. 192, no. 5-6, pp. 709-722, 2003.

[6] L. Marin and D. Lesnic, "The method of fundamental solutions for the Cauchy problem associated with two-dimensional Helmholtz-type equations," Computers and Structures, vol. 83, no. 4-5, pp. 267-278, 2005.

[7] L. Marin, L. Elliott, P. J. Heggs, D. B. Ingham, D. Lesnic, and $\mathrm{X}$. Wen, "Comparison of regularization methods for solving the Cauchy problem associated with the Helmholtz equation," International Journal for Numerical Methods in Engineering, vol. 60, no. 11, pp. 1933-1947, 2004.

[8] L. Marin, L. Elliott, P. J. Heggs, D. B. Ingham, D. Lesnic, and $\mathrm{X}$. Wen, "BEM solution for the Cauchy problem associated with Helmholtz-type equations by the Landweber method," Engineering Analysis with Boundary Elements, vol. 28, no. 9, pp. 1025-1034, 2004.

[9] T. Regińska and A. Wakulicz, "Wavelet moment method for the Cauchy problem for the Helmholtz equation," Journal of Computational and Applied Mathematics, vol. 223, no. 1, pp. 218229, 2009.

[10] H.-H. Qin and T. Wei, "Quasi-reversibility and truncation methods to solve a Cauchy problem for the modified Helmholtz equation," Mathematics and Computers in Simulation, vol. 80, no. 2, pp. 352-366, 2009.

[11] X. L. Feng, C. L. Fu, and H. Cheng, "A modified Tikhonov regularization for solving the Cauchy problem for the Helmholtz equation," http://www.mai.liu.se/xifen.

[12] H. H. Qin, T. Wei, and R. Shi, "Modified Tikhonov regularization method for the Cauchy problem of the Helmholtz equation," Journal of Computational and Applied Mathematics, vol. 224, no. 1, pp. 39-53, 2009.

[13] C.-L. Fu, X.-L. Feng, and Z. Qian, "The Fourier regularization for solving the Cauchy problem for the Helmholtz equation," Applied Numerical Mathematics, vol. 59, no. 10, pp. 2625-2640, 2009.

[14] T. Regińska and K. Regiński, "Approximate solution of a Cauchy problem for the Helmholtz equation," Inverse Problems, vol. 22, no. 3, pp. 975-989, 2006.

[15] T. Regińska and U. Tautenhahn, "Conditional stability estimates and regularization with applications to cauchy problems for the helmholtz equation," Numerical Functional Analysis and Optimization, vol. 30, no. 9-10, pp. 1065-1097, 2009.

[16] H. Cheng and C.-L. Fu, "An iteration regularization for a timefractional inverse diffusion problem," Applied Mathematical Modelling, vol. 36, no. 11, pp. 5642-5649, 2012.

[17] H. W. Engl, M. Hanke, and A. Neubauer, Regularization of Inverse Problems, Kluwer Academic, Boston, Mass, USA, 1996.

[18] M. T. Nair, E. Schock, and U. Tautenhahn, "Morozov's discrepancy principle under general source conditions," Zeitschrift für Analysis und ihre Anwendungen, vol. 22, no. 1, pp. 199-214, 2003. 


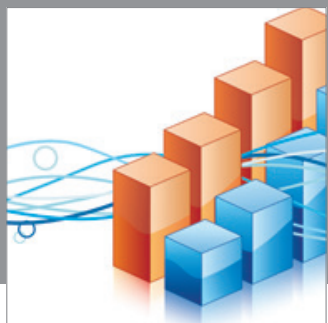

Advances in

Operations Research

mansans

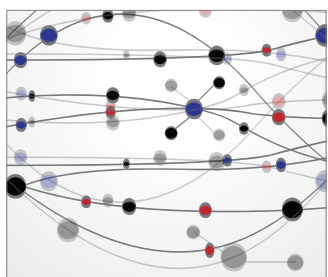

The Scientific World Journal
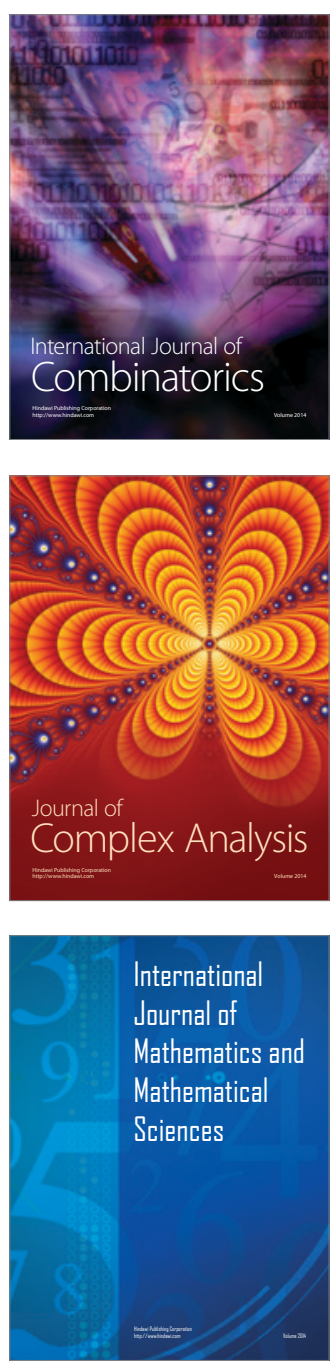
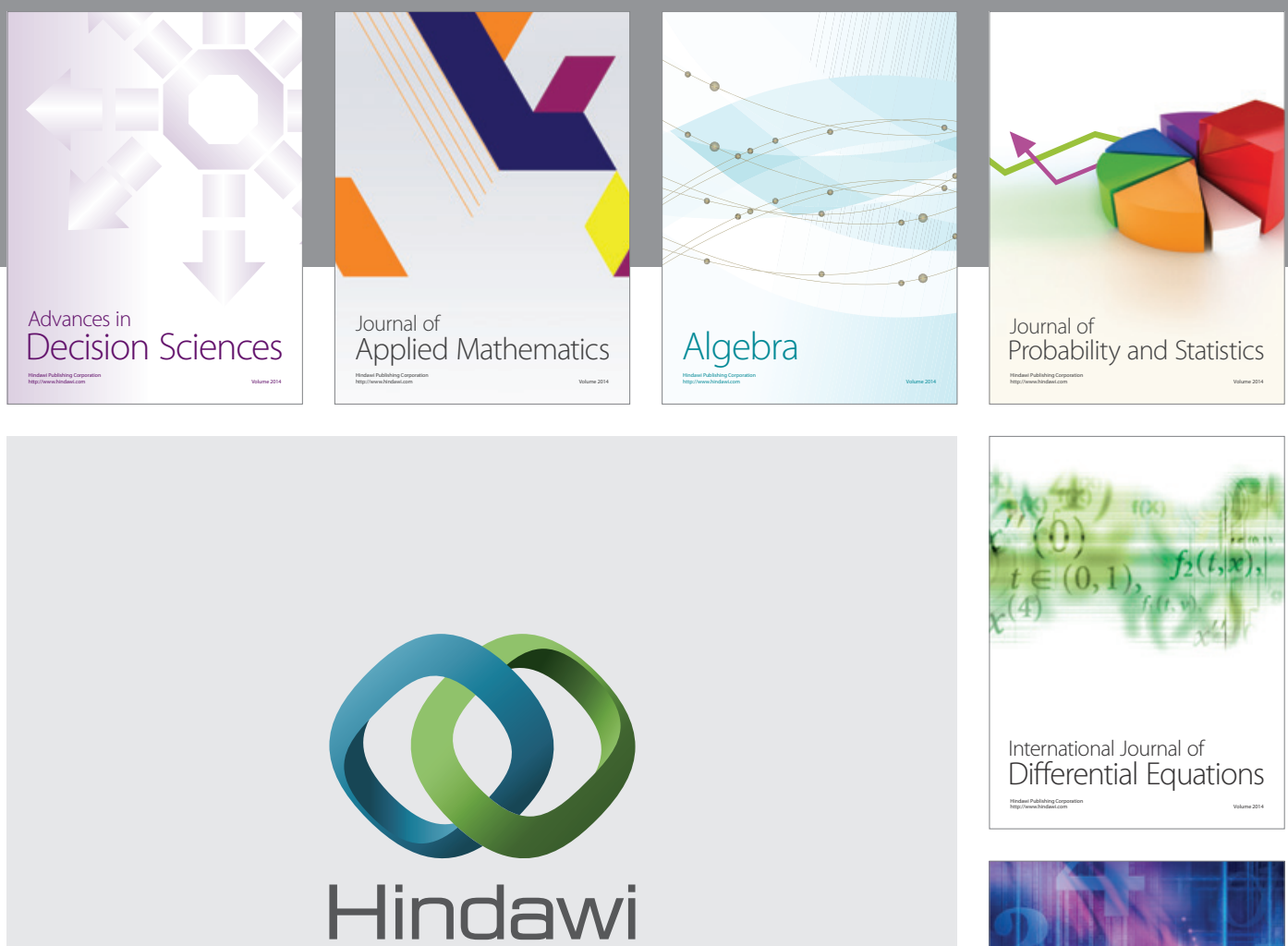

Submit your manuscripts at http://www.hindawi.com
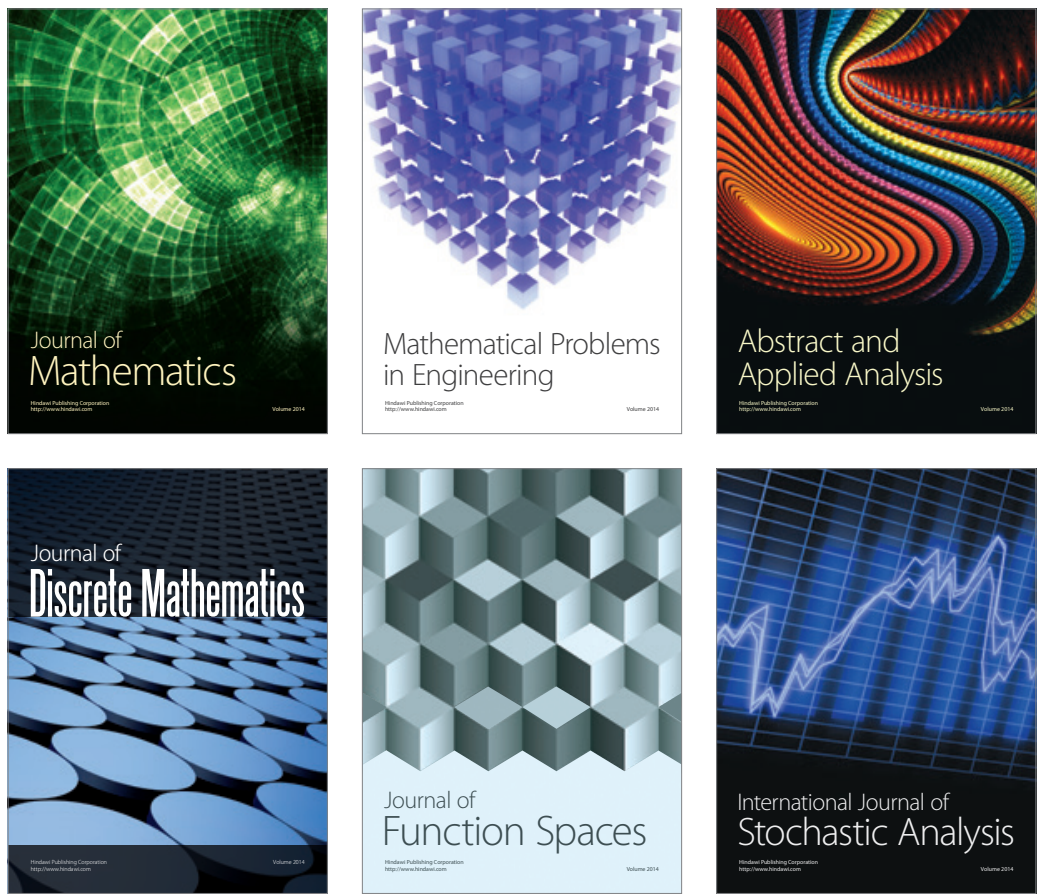

Journal of

Function Spaces

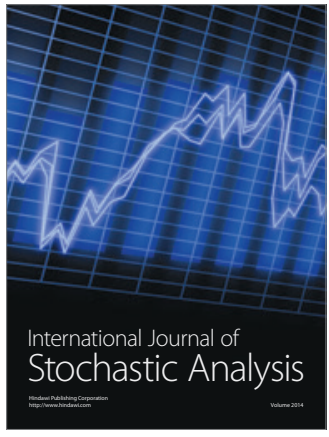

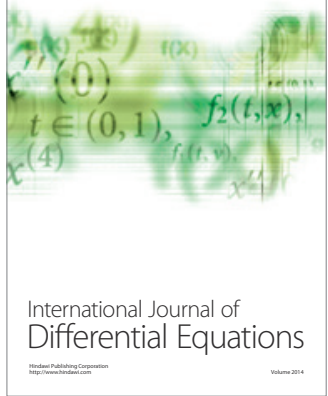
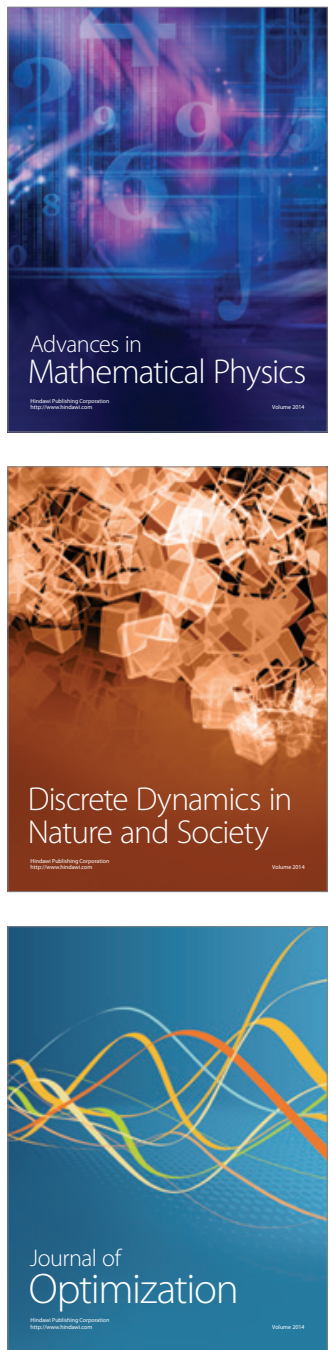